\title{
CONGRUENCE LATTICES OF SMALL PLANAR LATTICES
}

\author{
G. GRÄTZER, H. LAKSER, AND E. T. SCHMIDT
}

\begin{abstract}
For a finite distributive lattice $D$ with $n$ join-irreducible elements, we construct a finite (planar) lattice $L$ with $O\left(n^{2}\right)$ elements such that the congruence lattice of $L$ is isomorphic to $D$. This improves on an early result of R. P. Dilworth (around 1940) and G. Grätzer and E. T. Schmidt (1962) constructing such a (nonplanar) lattice $L$ with $O\left(2^{2 n}\right)$ elements, and on a recent construction of $\mathrm{G}$. Grätzer and $\mathrm{H}$. Lakser which yields a finite (planar) lattice $L$ with $O\left(n^{3}\right)$ elements.
\end{abstract}

\section{INTRODUCTION}

A classical result of R. P. Dilworth (circa 1940, unpublished, see [1], pp. 455457) represents a finite distributive lattice $D$ as the congruence lattice of a finite lattice $L$. The first published proof of this result by G. Grätzer and E. T. Schmidt [7] constructed a finite lattice $L$ with $O\left(2^{2 n}\right)$ elements, where $n$ is the number of join-irreducible elements of $D$.

Curiously, the construction technique developed by G. Grätzer and H. Lakser [4] to construct a complete lattice with a given (complete) lattice of complete congruences helped the same authors [6] to construct a finite lattice $L$ with $O\left(n^{3}\right)$ elements; this lattice $L$ is also planar.

In this paper, we improve this result by constructing a finite (planar) lattice $L$ with $O\left(n^{2}\right)$ elements.

Theorem . Let $D$ be a finite distributive lattice with $n$ join-irreducible elements. Then there exists a lattice $L$ with $O\left(n^{2}\right)$ elements whose congruence lattice is isomorphic to $D$. This lattice $L$ can be chosen to be planar.

For the basic concepts of lattice theory, we refer the reader to [2].

\section{The Construction}

Let $D$ be a finite distributive lattice, and let $J=J(D)$ be the poset of its join-irreducible elements. Let $n$ be the cardinality of $J$.

A coloring of a finite chain $C$ is a map of the prime intervals of $C$ into $J$.

Let $J_{0}=\left\{a_{0}, a_{1}, \ldots, a_{k-1}\right\}$ be the set of nonminimal elements of $J$. If $k=0$, then $J$ is unordered, and we can choose $L$ as a chain of length $n$. Henceforth, we assume that $k>0$.

Date: June 6, 1993.

1991 Mathematics Subject Classification. Primary 06B10; Secondary 06D05.

Key words and phrases. Lattice, finite, congruence, distributive, planar.

The research of the first two authors was supported by the NSERC of Canada.

The research of the third author was supported by the Hungarian National Foundation for Scientific Research, under Grant No. 1903.

The result of this paper was announced at the Bjarni Jónsson Symposium in Iceland (1990). See also Abstracts of papers presented to the Amer. Math. Soc. 90, p. T-06-187. 
Let $C_{0}$ be a chain of length $2 k$. We color the prime intervals of $C_{0}$ as follows: we color the lowermost two prime intervals of $C_{0}$ with $a_{0}$, the next two with $a_{1}$, and so on. Thus, for each $a \in J_{0}$, there are in $C_{0}$ two prime intervals of color $a$ (and they are successive). So for each $a \in J_{0}$, there is a unique subchain $a^{b} \prec a^{m} \prec a^{t}$ such that the prime intervals $\left[a^{b}, a^{m}\right]$ and $\left[a^{m}, a^{t}\right]$ have color $a$, and no other prime interval has color $a$. Observe that, for each $a$ distinct from $a_{0}, a^{b}=c^{t}$ for some $c \in J_{0}$, and, similarly, for each $a$ distinct from $a_{k-1}, a^{t}=c^{b}$ for some $c \in J_{0}$. The elements $a^{m}$, however, are labelled uniquely.

Let $C_{1}$ be a chain of length $n=|J|$. We color the prime intervals of $C_{1}$ by an arbitrary bijection. Thus, for each $a \in J$, there is in $C_{1}$ exactly one prime interval of color $a$; we denote it by $\left[a^{\circ}, a^{i}\right]$.

We set $L_{0}=C_{0} \times C_{1}$. We shall regard $C_{0}$ and $C_{1}$ as sublattices of $L_{0}$ in the usual manner.

The poset of join-irreducible congruences of a finite lattice $L$ can be represented by the prime intervals of $L$ as follows (see, e.g., [2]). Let $\mathfrak{p}$ and $\mathfrak{q}$ be prime intervals of $L$; let $\mathfrak{p} \leq \mathfrak{q}$ mean that $\mathfrak{p}$ is weakly projective into $\mathfrak{q}$. This defines a quasiordering on the set of prime intervals of $L$. Regard $\mathfrak{p}$ and $\mathfrak{q}$ equivalent if $\mathfrak{p} \leq \mathfrak{q}$ and $\mathfrak{q} \leq \mathfrak{p}$. Then the relation $\leq$ defines a partial ordering on the set of equivalent prime intervals. This partially ordered set represents the poset of join-irreducible congruences.

In $L_{0}$, we can choose the prime intervals in $C_{0} \cup C_{1}$ as representatives of the equivalence classes. Hence, the poset of join-irreducible congruences of $L_{0}$ is a totally unordered poset of cardinality $2 k+n$.

Note that

$$
\left|L_{0}\right|=(2 k+1)(n+1) \text {. }
$$

We next extend the lattice $L_{0}$ to a lattice $L_{1}$. For each $a \in J_{0}$ we adjoin two new elements $m_{0}(a)$ and $m_{1}(a)$ to $L_{0}$; we set

$$
\left\langle a^{b}, a^{o}\right\rangle \prec m_{0}(a) \prec\left\langle a^{m}, a^{i}\right\rangle
$$

and

$$
\left\langle a^{m}, a^{\circ}\right\rangle \prec m_{1}(a) \prec\left\langle a^{t}, a^{i}\right\rangle
$$

See Figure 1; the new elements are black-filled. The resulting poset $L_{1}$ is a lattice, and, for each $a \in J_{0}$, the intervals

$$
\left[\left\langle a^{b}, a^{o}\right\rangle,\left\langle a^{m}, a^{i}\right\rangle\right]=\left\{\left\langle a^{b}, a^{o}\right\rangle,\left\langle a^{m}, a^{o}\right\rangle, m_{0}(a),\left\langle a^{b}, a^{i}\right\rangle,\left\langle a^{m}, a^{i}\right\rangle\right\}
$$

and

$$
\left[\left\langle a^{m}, a^{o}\right\rangle,\left\langle a^{t}, a^{i}\right\rangle\right]=\left\{\left\langle a^{m}, a^{o}\right\rangle,\left\langle a^{t}, a^{o}\right\rangle, m_{1}(a),\left\langle a^{m}, a^{i}\right\rangle,\left\langle a^{t}, a^{i}\right\rangle\right\}
$$

are isomorphic to the five-element modular nondistributive lattice $\mathbf{M}_{3}$.

By adjoining these elements, we have made equivalent any two prime intervals of $L_{1}$ in $C_{0} \cup C_{1}$ of the same color. Therefore, the poset of join-irreducible congruences of $L_{1}$ is isomorphic to the poset $J$ with the discrete partial order.

Observe that

$$
\left|L_{1}\right|=(2 k+1)(n+1)+2 k \text {. }
$$

We finally further extend $L_{1}$ so as to induce the correct partial order on the joinirreducible congruences. For each pair $a \succ c$ in $J$ (whereby $a \in J_{0}$, necessarily), we 
add a new element $n(a, c)$ to $L_{1}$, setting

$$
\left\langle a^{m}, c^{\circ}\right\rangle \prec n(a, c) \prec\left\langle a^{m}, c^{i}\right\rangle,
$$

as illustrated in Figure 2. The resulting poset $L$ is a lattice. In $L$, the interval $\left[a^{m}, a^{t}\right]$ is projective into $\left[n(a, c),\left\langle a^{m}, c^{i}\right\rangle\right]$, which in turn is projective into $\left[c^{o}, c^{i}\right]$. So the poset of join-irreducible congruences of $L$ is isomorphic to $J$. Consequently, the congruence lattice of $L$ is isomorphic to $D$.

Note that $L$ is a planar lattice, and that in going from $L_{1}$ to $L$ we adjoin no more than $k n$ elements. Thus

$$
|L| \leq(2 k+1)(n+1)+2 k+k n<3(n+1)^{2} .
$$

\section{Comments}

The construction in [6] utilized the first chain to establish the join-irreducible congruences and to order them. The second chain served only one purpose: to help identify the congruences generated by different prime intervals of the same color.

We achieve greater economy in the present construction because the second chain serves the additional purpose of ordering the congruences generated by the prime intervals of the first chain. As a result, it is enough to have at most two prime intervals of the same color in the first chain.

A formal proof of the Theorem will not be given because the construction is so intuitively clear. A formal proof would associate with $a \in J$ the congruence $\Theta_{a}^{0}$ on $L_{0}$ generated by the three prime intervals of color $a$ of $C_{0} \cup C_{1}$. Then it would be verified that $\Theta_{a}^{0}$ extends from $L_{0}$ to the congruence $\Theta_{a}^{1}$ of $L_{1}$, and $\left\{\Theta_{a}^{1} \mid a \in J\right\}$ is the (unordered) set of join-irreducible congruences of $L_{1}$. Finally, it would be verified that $\Theta_{a}^{1}$ extends from $L_{1}$ to the congruence $\Theta_{a}$ of $L$, and $a \rightarrow \Theta_{a}$ is an isomorphism between $J$ and the partially ordered set of join-irreducible congruences of $L$.

The congruence extension statements are trivial directly; or it could be pointed out that the first can be obtained by applying the Colored Product Extension Theorem (Theorem 7 of [5]), and the second by the One Point Extension Theorem (Theorem 6 of $[5]$ ).

\section{REFERENCES}

[1] K. P. Bogart, R. Freese, and J. P. S. Kung (editors), The Dilworth Theorems. Selected papers of Robert P. Dilworth, Birkhäuser Verlag, Basel-Boston, 1990, pp. 460-464.

[2] G. Grätzer, General Lattice Theory, Pure and Applied Mathematics Series, Academic Press, New York, 1978; Mathematische Reihe, Band 52, Birkhäuser Verlag, Basel; Akademie Verlag, Berlin.

[3] G. Grätzer, Results on the congruence lattice of a lattice, in The Dilworth Theorems. Selected papers of Robert P. Dilworth (K. P. Bogart, R. Freese, and J. P. S. Kung eds.), Birkhäuser Verlag, Basel-Boston, 1990, pp. 460-464.

[4] G. Grätzer and H. Lakser, On complete congruence lattices of complete lattices, Trans. Amer. Math. Soc. 327 (1991), pp. 385-405.

[5] G. Grätzer and H. Lakser, On congruence lattices of $\mathfrak{m}$-complete lattices, J. Australian Math. Soc. (Series A) 52 (1992), pp. 57-87.

[6] G. Grätzer and H. Lakser, Congruence lattices of planar lattices, Acta Math. Hungar. 60 (1992), pp. 251-268.

[7] G. Grätzer and E. T. Schmidt, On congruence lattices of lattices, Acta Math. Acad. Sci. Hungar. 13 (1962), pp. 179-185. 
Defartment of Mathematics, University of Manitoba, Winnifeg, Man. R3T 2N2, CANADA

E-mail address: George_Gratzer@umanitoba.ca

E-mail address: hlakser@ccu.umanitoba.ca

Department of Mathematics, Transfort Engineering Faculty, Technical University of Budapest, Müegyetem RKf. 9, 1111 Budapest, Hungary

E-mail address: h1175sch@ella.hu 